\title{
Validation of a new non-invasive blood pressure measurement method on mice via pulse wave propagation time measurement on a cuff
}

\author{
Xuan P. Nguyen ${ }^{1, *}$, Ralf Kronemayer ${ }^{1}$, Peter \\ Herrmann $^{2}$, Atila Mejía ${ }^{1}$, Zamira Daw ${ }^{1}$, Xuan D. \\ Nguyen $^{3}$, Bettina Kränzlin ${ }^{4}$ and Norbert Gretz ${ }^{4}$ \\ ${ }^{1}$ Mannheim Biomedical Engineering Laboratories \\ (MABEL), University Hospital Mannheim LiMedical \\ Faculty Mannheim, Heidelberg University, Germany \\ ${ }^{2}$ Department of Anesthesiology, Emergency and Intensive \\ Care Medicine, University of Göttingen, Germany \\ ${ }^{3}$ Institute of Transfusion Medicine and Immunology, \\ Medical Faculty Mannheim, Heidelberg University, \\ German Red-Cross Blood Service of Baden-Württemberg, \\ Hessen, Germany \\ ${ }^{4}$ Medical Research Center, University Hospital Mannheim, \\ Medical Faculty Mannheim, Heidelberg University, \\ Germany
}

\begin{abstract}
In the present article, we describe the validation of a new non-invasive method for measuring blood pressure (BP) which also enables to determine the three BP values: systolic, diastolic and mean value. Our method is based on the pulse transit time (PTT) measurement along an artery directly at the BP cuff. The accuracy of this method was evaluated by comparison with the direct simultaneous measurement of blood pressure from 40 anesthetized female mice. Close correlation between the gained data from these two methods was observed.
\end{abstract}

Keywords: diastolic pressure; direct blood pressure; mean pressure; non-invasive blood pressure measurement; pulse transit time; systolic arterial pressure.

\section{Introduction}

Mice as laboratory test animals play a significant and vital role in the medical research conducted. Therefore, the physiological parameters of the mice, BP and heart rate, need to be characterized.

\footnotetext{
*Corresponding author: Dr. sc. hum. Xuan Phuc Nguyen, Mannheim Biomedical Engineering Laboratories, Mannheim D-68163, John-Deere-Straße 85 Paul Wittsack, Str. 10. D-68163 Mannheim, Germany

Phone: +496212926294

Fax: +49621 2926454

E-mail: p.nguyen@hs-mannheim.de
}

The BP can be measured in an invasive or non-invasive way. The non-invasive way measurement is substantially more suitable than the invasive method for investigations where there is a mortality risk and possible health complications, and the life of the test animal must be preserved. Various methods of indirect measurement of arterial blood pressure in mice have been described [2, 3, 7, 14, 16]. In most of these methods, blood pulse or blood volume variations corresponding to the systolic end point are detected. These methods measure exclusively the systolic pressure. Other methods, based on the vascular unloading (Penàz method) technique [16] and the volume-pressure recording [4], are more suitable to measure the blood pressure in a continuous way. Although some studies have suggested that there is reasonable accuracy with these methods, the technology itself is generally expensive.

An alternative for monitoring the blood pressure with the passive non-invasive method is available with PTT measurements. However, this technique has found limited use due to inherent drawbacks in the existing technology. The electrocardiogram $\mathrm{R}$ wave is often used as a proximal timing point since it is simple to detect and tolerant of motion artifact. However, there is a considerable delay between the onset of electrical cardiac activity and the start of mechanical ventricular ejection. It is referred to as the pre-ejection period. Late studies, however, demonstrate that the use of electrocardiogram R wave purely for the assessment of the PTT is inappropriate and should be avoided since the pre-ejection period cannot be assumed to remain constant [13].

In this study, we describe a novel blood pressure monitor based on pulse wave velocity. This monitor consists of two photoplethysmography sensors in line. The second photoplethysmography sensor eliminates the need for the electrocardiogram and allows defining in just one procedure the three main features of the BP signals: systolic (SBP), mean (MBP) and diastolic (DBP). The importance of the three measurements is that the systolic and diastolic pressure represents the healthy state of the heart and the arteries, while the mean pressure shows the physiological characteristics of the left ventricular contractility, vascular resistance and elasticity averaged over time [6].

\section{Methods}

\section{Subjects and materials}

Measurements were conducted with a total of 20 female Naval Medical Research Institute (NMRI) and Fibroblast 


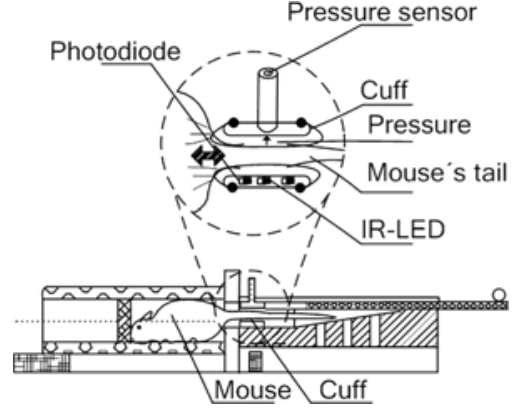

Figure 1 Schematic diagram for indirect measurement of mouse blood pressure.

Growth Factor (FGF) mice. A heterogeneous selection of mice (different age, weight and genealogy) was taken into account in order to validate the new method without requiring drugs for a specific increase or reduction of BP [8-10].

Figure 1 illustrates a schematic diagram of the device. The system consists of two photodiodes a separated a minimum $15 \mathrm{~mm}$ from each other. This distance is based on the minimum detectable PTT of our system ( $3 \mathrm{~ms}$ ) and the maximal pulse wave velocity reported in mice $(4.60 \mathrm{~m} / \mathrm{s})$ [5]. They record the reflected light of an infrared LED attached to the tail of the mice. At the same time, a pressure cuff is initially inflated to a pressure $(180 \mathrm{~mm} \mathrm{Hg})$ that exceeds the systolic arterial pressure of the mice. It is then reduced by a rate of approximately $2.5 \mathrm{~mm} \mathrm{Hg} / \mathrm{s}$.

The recorded signals of the photodiodes are then used to estimate the PTT curve, this curve will be later correlated with the recorded signal of the cuff pressure. The method described in this paper can also be implemented in a trans- mission mode if the distance between the light source and the sensors is smaller than $1 \mathrm{~cm}$.

\section{Principle}

The result of the external pressure applied to the artery by the cuff is the modulation of the artery characteristics such as the compliance. This modulation can be described through the formula $(1)[11,15]$.

$C\left(P_{i}, P_{\text {cuff }}\right)=\frac{R_{\max }^{2}}{P_{1}}\left(\frac{1}{1+\left(\frac{P_{i}-P_{\text {cuff }}}{P_{1}}\right)^{2}}\right)$

$P_{1}$ : relates to the slope at the inflection point,

$P_{i}$ : internal pressure, $P_{\text {cuff }}=$ cuff pressure,

$R_{\max }$ : is the maximal asymptotic vessel radius.

Based on the Bramwell-Hill formula (2) can be demonstrated the relation between the PTT, the compliance, and cuff pressure [1].

$v=\frac{d}{P T T}=\sqrt{\frac{1}{\rho C\left(P_{i}, P_{c u f f}\right)}}$

$\rho$, blood density; $d$, distance between sensors.

Figure 2 shows the relation between the PTT and compliance. The three BP values are indicated.

The method used in this study takes advantage of this effect in order to estimate the BP indirectly. The PTT is calculated between the recorded signals of the photodiodes. It is obtained from the sensor nearest to the heart (proximal) and the signal of the sensor situated further away from the heart (distal). It shows the three characteristic features for

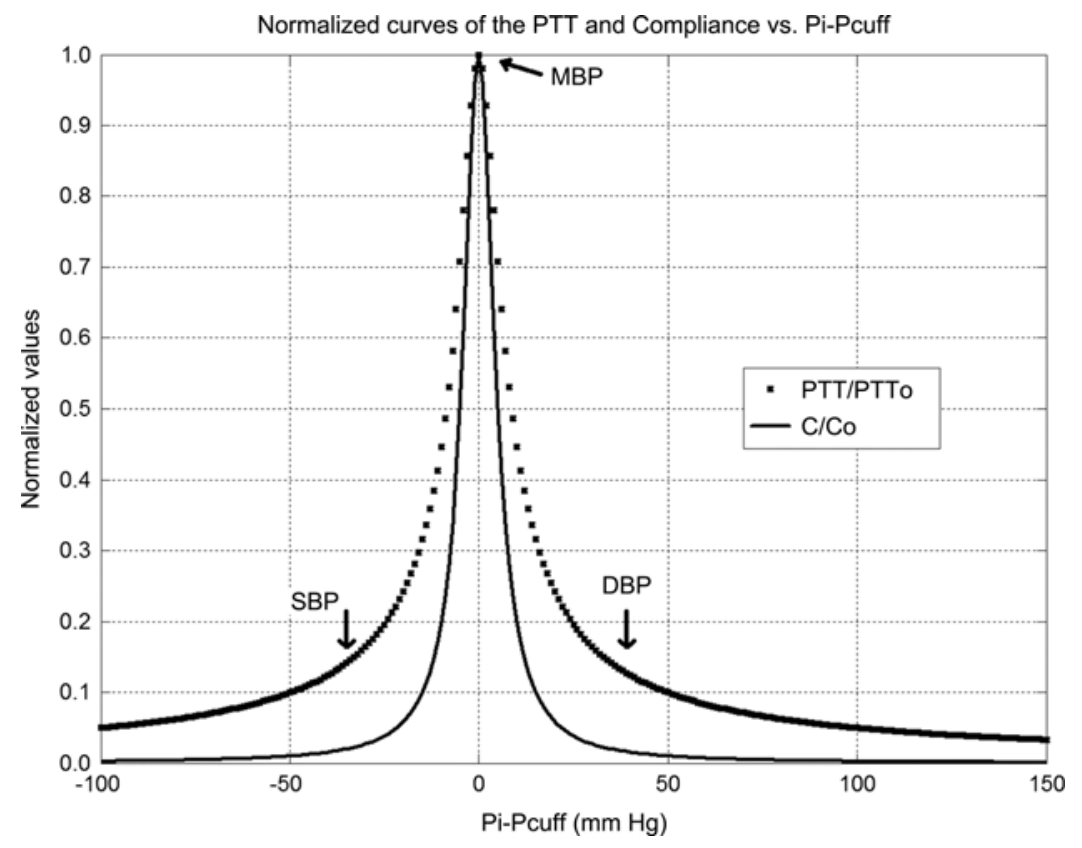

Figure 2 Modulation of the artery characteristics: compliance and PTT as function of the internal and the cuff pressure. It shows too the three BP values. 


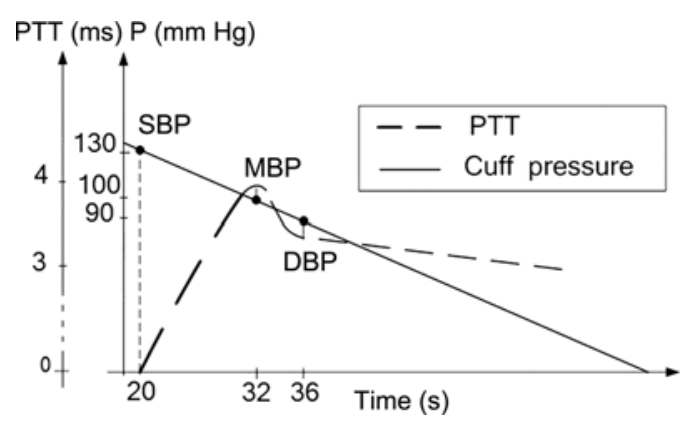

Figure 3 Characteristic points in the PTT curve. The descending curve shows the cuff pressure.

the determination of the systolic, the mean and the diastolic BP (Figure 3). The following paragraph explains the method developed on which we based this study. There are four phases:

- The cuff is initially inflated to a pressure of $180 \mathrm{~mm} \mathrm{Hg}$ that exceeds the systolic arterial pressure of the mouse. As a result of the external pressure, the artery is totally compressed and therefore the blood cannot circulate and thus the pulse cannot be detected. The cuff pressure is reduced by a rate of approximately $2.5 \mathrm{~mm} \mathrm{Hg} / \mathrm{s}$.

- At the time at which the cuff pressure is the same as the systolic BP, the blood can circulate again and the wave appears distal of the cuff, the transit time is at its minimum point. The increase of the transit time curve is considered a characteristic feature.

- Maximum of the transit time curve. At this point, the cuff pressure is equal to the mean BP. Due to this pressure; the compliance of the artery is maximal. Hence, the pulse wave velocity is minimal while the PTT is maximal [17].

- Once the compliance reaches the maximal point it starts to decrease and with it the PTT. When the cuff pressure is the same as the diastolic value, the modulation of the artery by the cuff is completed and the compliance remains constant. The buckling in the PTT curve is the diastolic BP.

\section{Experiments with the mice}

The measurements were approved by the District Governmental Commission on the Care and Use of Animals. The mice were anesthetized with Ketamin/Xylazin $(120 \mathrm{mg} /$ $16 \mathrm{mg}$ per kg weight) intravenously. The arterial carotid pressure was measured directly using a Samba micro-pressure sensor (SE-421 30, Samba Sensors AB, Västra Frölunda, Sweden) and an indwelling arterial catheter (PolyethyleneCatheter O.D.: $0.61 \mathrm{~mm}$, I.D.: $0.28 \mathrm{~mm}$, Becton-Dickinson, Heidelberg, Germany) that was carefully introduced, at maximum $0.5 \mathrm{~cm}$ into the vessel, in order to prevent entering the heart region. With the help of a trocar, the catheter became subcutaneously tunnelled directly to the neck area. The resigning end of the catheter was wrapped in fabric tape and was fixed with the help of a purse-string suture (Ethibond, $6-0$, Ethicon). The transaction in the neck region was sewn in (Ethibond, 6-0, Ethicon). To determine the normalized

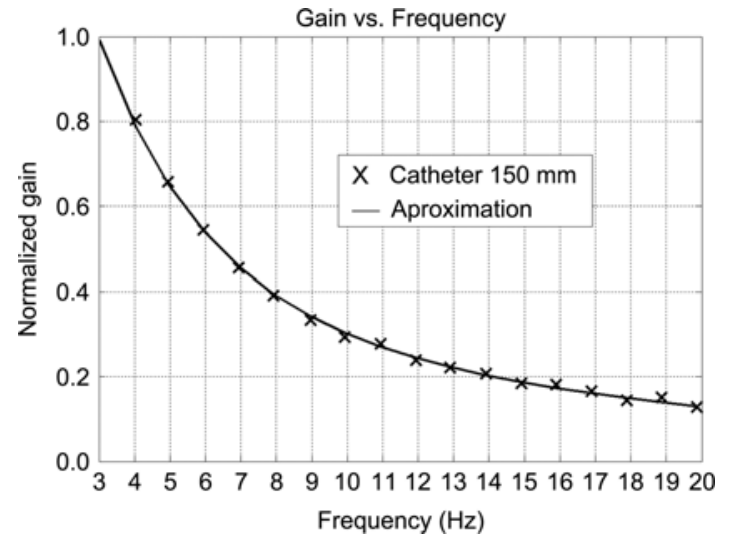

Figure 4 Pressure attenuation of the catheter $(150 \mathrm{~mm})$ in dependence of the frequency.

damping $(D)$ of the pressure pulse obtained by the cathetertransducer system as a function of the frequency, a first pressure sensor (Medex Transducer, Smiths Medical, London, UK) was connected directly to the pressure generator in order to register the pressure practically without attenuation. A second sensor the Samba - micro pressure sensor was connected via a catheter to the pressure-generator, the pressure generator performed a frequency sweep between 0 and $20 \mathrm{~Hz}$. The frequency dependence (Figure 4) can be described by the following formula:

$D(f)=a \cdot e^{b \cdot f}+c \cdot e^{d \cdot f}$

with $\mathrm{a}=1.6640, \mathrm{~b}=-0.3331, \mathrm{c}=0.4582$ and $\mathrm{d}=-0.0641$.

The formula was generated with the Matlab's curve fitting tool by which the constants $\mathrm{a}, \mathrm{b}, \mathrm{c}, \mathrm{d}$ are determined.

Hence, it is necessary to implement a correction factor in order to compare the direct and indirect measurements. For this reason the heart rate was estimated using the first harmonic frequency from the fast Fourier transform analysis of the proximal photodiode signal and this value is used to calculate the damping. To compensate the damping is used in the following formula:

direct $B P_{\text {compensated }}=\frac{\text { direct } B P_{\text {measured }}}{D(\text { heart rate })}$

This compensation was made only for the pulsatile components of the direct BP measurements (DBP and SBP) and not for the steady components (MBP), because the catheter damping in the range of the steady components does not affect the measurements as can be seen in the Figure 3.

During the operation and the recovery phase, the body temperature of the animals remained constantly at $36^{\circ} \mathrm{C}$ using special heating plates. The first measurement of the direct BP and the PTT were performed at the same time after the mice were woken up and after a relaxation time of at least $2 \mathrm{~h}$ post operation. The mice were placed in a rodent holder (Mouse rodent holder Model 84, IITC, California) so that the catheter could be easily accessed from outside. 
Once the photodiode signals and the cuff pressure signal were recorded, the pulses of the proximal and distal signals were detected. Each pulse was found by the identification of the maximal positive slope points of the signals. The PTT curve was calculated as the time difference between the corresponding detected points of the proximal and distal signals.

\section{Results}

$6 \pm 2$ measurements were carried out per mouse. Figure 5 shows a typical change of the transit time compared to a simultaneous direct BP measurement during the deflation phase of the cuff. The calculation of the indirect BP is based on the beat to beat measurement of the transit time and the locations of the three characteristic features: systolic, diastolic and mean blood pressures.

Figure 6 shows an example of the recordings of the proximal and distal signals (A), the PTT curve as a result of the time difference between both signals with the corresponding BPs (B) and the cuff pressure (C).

In Figure 7, the data of systolic, diastolic and mean blood pressures are summarized using a regression method $(5 \mathrm{~B}$, $5 \mathrm{D}, 5 \mathrm{~F})$ and the Bland-Altman plot $(5 \mathrm{~A}, 5 \mathrm{C}, 5 \mathrm{E})$ which is a statistical method to compare two measuring techniques. In the Bland-Altman plot, mean and standard deviation are calculated for the present system vs. the direct method. Table 1

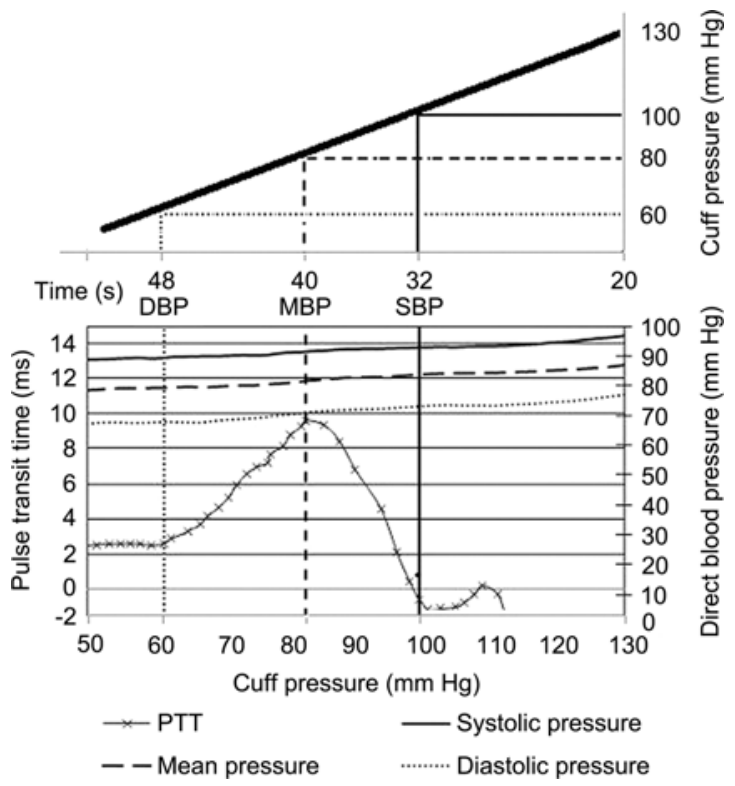

Figure 5 Typical PTT alterations $(*)$ during a deflation phase of the cuff (X-axis) compared to the direct measurements (right $\mathrm{Y}$ axis). The vertical lines show the BP determined by the interpolation with the cuff pressure. At the $100 \mathrm{~mm} \mathrm{Hg}$ cuff pressure the blood could circulate and the transit time is at its minimum point, it is the systolic pressure. At the $80 \mathrm{~mm} \mathrm{Hg}$ cuff pressure, the pressure is equal to the mean pressure and the PTT curve has a maximum. At the $60 \mathrm{~mm} \mathrm{Hg}$ cuff pressure the PTT curve has a buckling and it is the diastolic pressure.
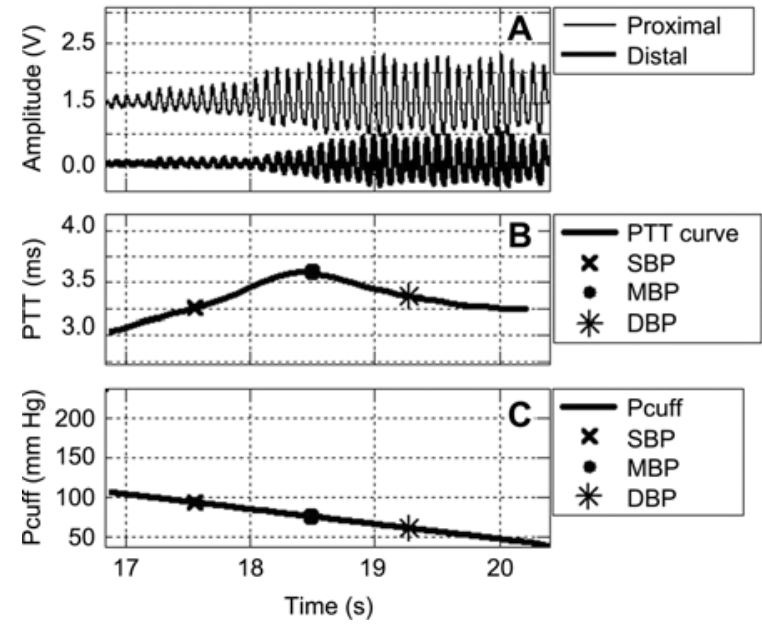

Figure 6 An example of the recorded distal and proximal signals (A). PTT curve with the SBP, BMP and DBP points (B). Cuff pressure curve with the corresponding BP points (C).

summarizes the comparison between the direct and the indirect measurements.

Value differences can be explained by the fact that there is a certain significant distance between the measuring positioning of the sensors and the heart. Therefore, the indirect systolic BP values are higher and the mean and diastolic BP values are lower than those of the direct measurements.

In order to be able to compare our measuring values to the values described in the literature, the average $\mathrm{BP}$ values from several measuring cycles of a session were calculated.

With this procedure, the comparison of the blood pressurevalue-pairs is more related to the mice than to the measuring cycles. Table 2 summarizes the comparison between the direct and the indirect measurements.

\section{Discussion}

In comparison to other methods, the novel indirect BP measuring method is at the moment the only non-invasive intermittent method which delivers three discrete characteristics for the identification of systolic, diastolic and mean blood pressures. Furthermore, this BP measuring method is based on a BP cuff which measures the BP intermittently.

The determination of the PTT along an arterial vessel directly at the BP cuff offers another big advantage: due to the physiology of the vessel, the cuff pressure dependent measurement of the transit time delivers three discrete characteristics to the determination of the systolic, mean and diastolic BP. Up until now this phenomenon has not been described in the literature.

The comparison of the two BP measurements, applying the Bland-Altman statistical method, shows that the results of the new BP measuring method agree with the directly measured BP. The relatively small standard-deviation of the diastolic and mean BP difference indicates that the charac- 

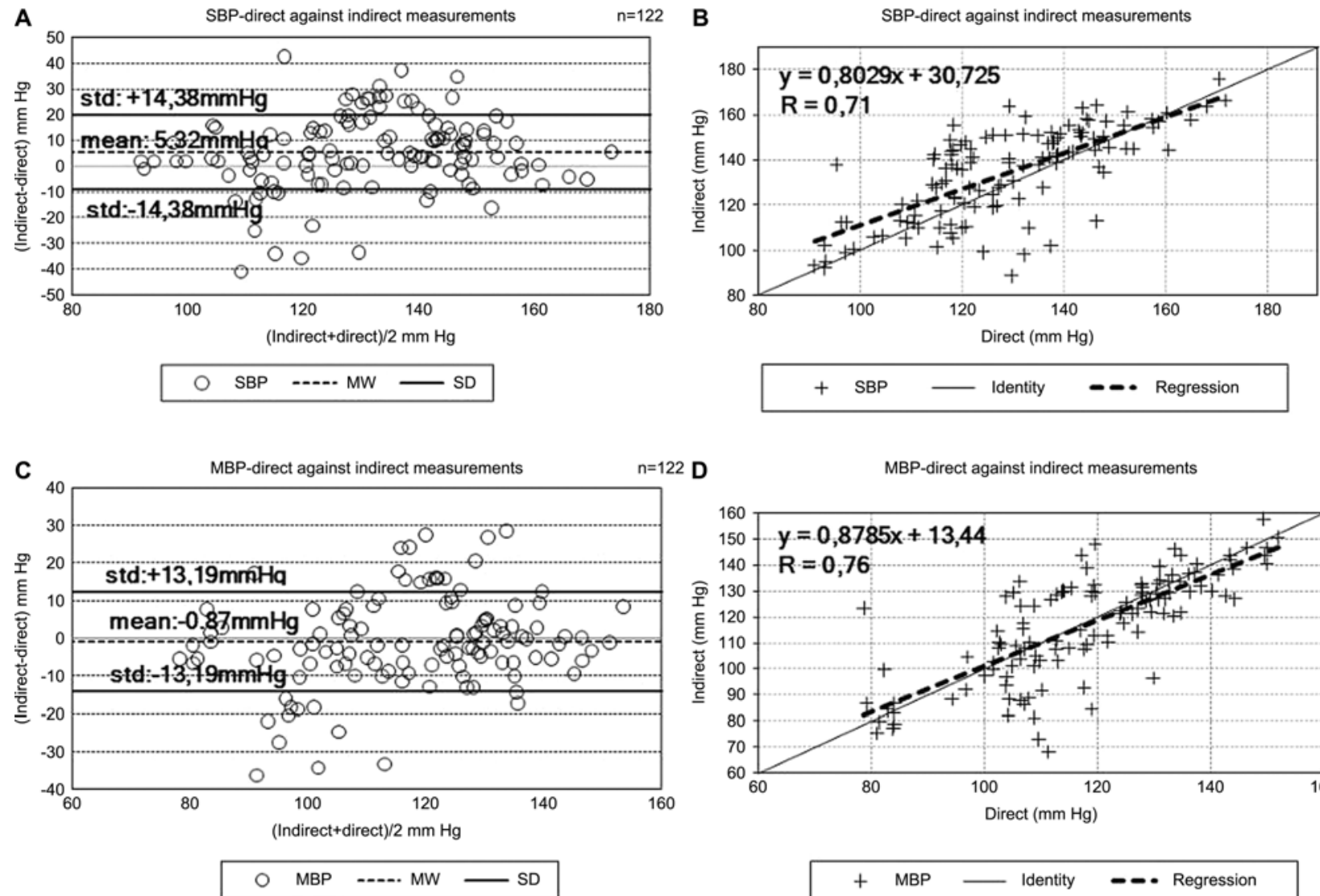

$=122 \mathrm{D}$
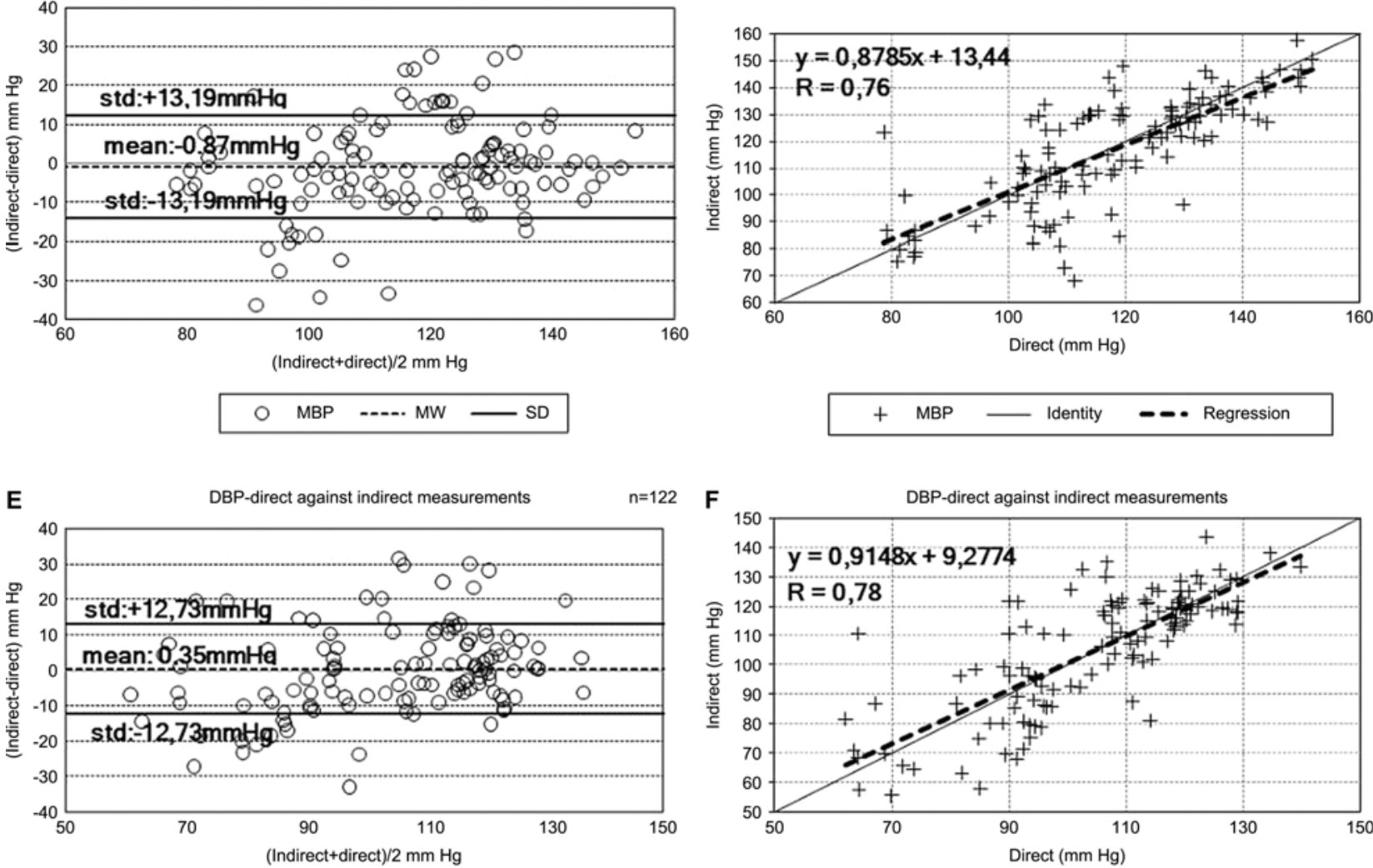

\begin{tabular}{llll}
\hline & DBP & $\cdots$ & MW
\end{tabular} SD

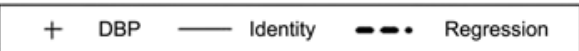

Figure 7 Indirect against indirect measurements: Systolic (SBP) (A, B), mean (MBP) (C, D), diastolic (DBP) (E, F) BP comparison to method of Bland and Altman (left) and to regression method (right) of all BP value-pairs bet.

$\mathrm{R}$, regression-coefficient; MW, blood pressure-difference; SD, standard-deviation.

Table 1 Statistical comparison between direct and indirect BP measurements (related to measuring cycles).

\begin{tabular}{lclll}
\hline BP & Difference & & p-Value & $\begin{array}{l}\text { Regression } \\
\text { coefficient }\end{array}$ \\
\cline { 2 - 3 } & Mean mm Hg & SD mm Hg & & \\
\hline SBP & 5.32 & 14.38 & 0 & 0.71 \\
MBP & -0.87 & 13.19 & 0 & 0.76 \\
DBP & 0.35 & 12.73 & 0 & 0.78 \\
\hline
\end{tabular}

Table 2 Statistical comparison between direct and indirect BP measurements (related to mice).

\begin{tabular}{llcll}
\hline BP & Difference & & p-Value & $\begin{array}{l}\text { Regression } \\
\text { coefficient }\end{array}$ \\
\cline { 2 - 3 } & Mean mm Hg & SD mm Hg & & \\
\hline SBP & 4.81 & 10.98 & 0 & 0.80 \\
MBP & 1.22 & 9.30 & 0 & 0.85 \\
DBP & 0.02 & 8.00 & 0 & 0.89 \\
\hline
\end{tabular}


teristics of the PTT curve are continuously stable. On the other hand, the comparisons of the systolic BP measuring values show a lower stability.

Measuring the cuff pressure by the systolic BP does not provoke a clear change in the transit time curve in some animals. As a result, the transit time gradient does not show a distinctive onset in each animal.

The most important factor influencing the accuracy of the measurement relies is the calculation of the PTT. It implies the pulse recognition in the distal and proximal photodiode signals and the time difference between the corresponding detected pulses. Taking into account that the maximal pulse velocity is $4.60 \mathrm{~m} / \mathrm{s}$, for short distances between sensors, the PTT has a magnitude of milliseconds. In order to improve the accuracy of the method it is recommended to increase the signal-to-noise ratio of the proximal and distal signals.

In conclusion, the proposed method has shown to be a useful tool for the indirect measurement of the arterial pressure in the mouse tail. In pursuance of the Langewouters study [12] there is similarities between the anatomy of the mouse tail and the human finger. With this premise, the proposed method seems to have a good perspective to find use in applications for humans.

\section{Acknowledgments}

The authors wish to thank MsC. Gustavo Guarín, MsC. Grace Villacrés, Mannheim Biomedical Engineering Laboratories (MABEL) and University Hospital Mannheim for their help in the review of this paper.

\section{References}

[1] Bramwell JC, Hill AV. The velocity of the pulse wave in man. Proc R Soc Lond B 1922; 93:298-306.

[2] Bunag RD. Validation in awake rats of a tail-cuff method for measuring systolic pressure. J Appl Physiol 1973; 34:279-282.

[3] Byrom FB, Wilson C. A plethysmographic method for measuring systolic blood pressure in the intact rat. J Physiol 1938; 93:301-304.

[4] Feng M, Whitesall S, Zhang Y, Beibel M, D'Alecy L, DiPetrillo K. Validation of volume-pressure recording tail-cuff blood pressure measurements. Am J Hypertens 2008; 21: $1288-1291$.
[5] Hartley CJ, Taffet GE, Michael LH, Pham TT, Entman ML. Noninvasive determination of pulse-wave velocity in mice. J Physiol Heart Circ Physiol 1997; 273:H494-H500.

[6] Howard DS, Meier JS, Bernard R. Systolic and diastolic blood pressure, pulse pressure, and mean arterial pressures as predictors of cardiovascular disease risk, in men. Hypertension, American Heart Association. 2000; 36:801-807.

[7] Ikeda K, Nara Y, Yamori Y. Indirect systolic and mean blood pressure determination by a new tail cuff method in spontaneously hypertensive rats. Lab Anim 1991; 25:26-29.

[8] Johns C, Gavras I, Handy DE, Salomao A, Gavras H. Models of experimental hypertension in mice. Hypertension 1996; 28:1064-1069.

[9] Krege JH, Hodgin JB, Hagaman JR, Smithies O. A noninvasive computerized tail-cuff system for measuring blood pressure in mice. Hypertension 1995; 25:1111-1115.

[10] Kurtz T, Griffin K, Bidani A, Davisson R, Hall J. Recommendations for blood pressure measurement in humans and experimental animals. Hypertension 2005; 45:299-310.

[11] Langewouters GJ, Wesseling KH, Goedhard WJ. A. The static elastic properties of 45 human thoracic and 20 abdominal aortas in vitro and the parameters of a new model. J Biomechanics 1984; 17:425-435.

[12] Langewouters GJ, Zwarti A, Busseg R, Wesselingt KH. Pressure-diameter relationships of segments of human finger arteries. Clin. Phys. Physiol. Meas. 1986; 7:43-55.

[13] Payne RA, Symeonides CN, Webb DJ, Maxwell SR. Pulse transit time measured from the ECG: an unreliable marker of beat-to-beat blood pressure. J Appl Physiol 2006; 100:136141.

[14] Pfeffer JM, Pfeffer MA, Frohlich ED. Validity of an indirect tail cuff method for determining systolic arterial pressure in anaesthetized normotensive and spontaneously hypertensive rats. J Lab Clin Med 1971; 78:957-962.

[15] Wesseling KH, Jansen JRC, Settels JJ, Schreuder JJ. Computation of aortic flow from pressure in humans using a nonlinear, three-element model. Modeling in Physiology. American Physiological Society 1993; 0161-7567/93:2566-2573.

[16] Yamakoshi KI, Shimazu H, Togawa T. Indirect measurement of instantaneous arterial blood pressure in the rat. Am J Physiol 1979; 237:632-637.

[17] Yamakoshi KI, Shimazu H, Shibata M, Kamiya A. New oscillometric method for indirect measurement of systolic and mean arterial pressure in the human finger. Med. Biol. Eng. Comput 1982; 20:307-313.

Received October 1, 2010; accepted April 19, 2011 